\title{
Numerical Simulation of Microstructure Evolution of Al Alloys in Centrifugal Casting
}

\author{
S. R. CHANG, J. M. KIM and C. P. HONG
}

Center for Computer-Aided Materials Processing (CAMP), Department of Metallurgical Engineering, Yonsei University, 134 Shinchon-dong, Seodaemun-ku, Seoul, 120-749 Korea. E-mail: hong@yonsei.ac.kr.

(Received on December 19, 2000; accepted in final form on March 1, 2001)

\begin{abstract}
A coupled stochastic model has been developed for the prediction of solidification grain structures in centrifugal casting. The present model consists of two schemes: the cellular automaton to simulate the evolution of solidification structure and the finite volume method to calculate the heat transfer. The present model has been applied to predict the evolution of solidification structures in centrifugal casting of Al alloys. The effects of mold rotation velocity, solute concentration, melt superheat and mold preheating temperature on solidification structures were investigated. In addition, the evolution of dendritic microstructures was also simulated using a modified cellular automaton model. The simulated results were in good agreement with those obtained experimentally.
\end{abstract}

KEY WORDS: centrifugal casting; solidification structure simulation; cellular automaton; control volume; finite volume method; primary columnar zone; equiaxed zone; secondary columnar zone, Al-Si alloy, Al-Cu alloy.

\section{Introduction}

The solidification structure of castings is of great importance because of its role on mechanical properties. A fine equiaxed grain structure is required in order to obtain homogeneous and isotropic mechanical properties. It is well known that the centrifugally cast ingots are usually characterized by 'banding' phenomenon in which the distinctive structural discontinuity is featured. ${ }^{1-3)}$ The casting parameters that influence solidification structures can include the mold rotation velocity, the mold dimension, the mold preheating temperature, the pouring temperature of molten melt, and the alloy composition etc. Some experimental studies have been carried out to investigate the relationship between macrostructural morphology and involved casting parameters. ${ }^{1,2)}$ However, the influences of casting parameters on solidification morphology and its theoretical and quantitative description are still far from clear understanding. Besides, it is also very important to understand the mechanism of grain structure formation in centrifugal casting in order to design optimum centrifugal casting conditions for obtaining a desired uniform structure.

Experiments are known to always suffer from the fact that a large number of experimental parameters must be carefully controlled simultaneously. Therefore, there is a considerable potentiality of computer simulation for this purpose. Over the last ten years, extensive efforts have been made to develop various kinds of deterministic and stochastic models to predict the evolution of microstructures in solidification of alloys. $^{4-10)}$ Compared with deterministic models, stochastic models have the advantages of depicting graphically the structure features including grain shape and size. Several studies based on the stochastic cellular automaton technique have been reported to simulate the solidification grain structures formed in various casting processes. ${ }^{11-13)}$ However, no work has reported the prediction of solidification structures in centrifugal casting.

The purpose of the current study is to develop a new, coupled cellular automaton (CA) with the finite volume method to predict the structural evolution of $\mathrm{Al}$ alloys in centrifugal casting. The effects of mold rotation velocity, solute content, melt superheat and mold preheating temperature on the solidification structures were investigated. The dendritic microstructure was also simulated using a modified cellular automaton (MCA) model. ${ }^{14)}$ The simulated results were compared with those obtained experimentally.

\section{Experimental Procedure}

Figure 1 shows the schematic diagram of a vertical centrifugal casting equipment. Binary $\mathrm{Al}-\mathrm{Si}$ and $\mathrm{Al}-\mathrm{Cu}$ alloys with various solute contents of $0.0,0.5,1.0$, and 2.0 mass $\%$ were prepared from high purity aluminum ( $>99.99$ mass $\%$ ), and master alloys for the addition of silicon and copper. The alloy was melted in a graphite crucible using a high frequency induction furnace. After reaching a required temperature, the melt was then poured into the steel mold with an inner diameter of $60 \Phi \mathrm{mm}$, an outer diameter of $100 \Phi \mathrm{mm}$ and a height of $120 \mathrm{~mm}$, which was preheated to a given temperature and rotated with various mold rotation velocities of $0,50,100,200$ and $400 \mathrm{rpm}$, respectively. The mold rotation velocity was measured by a tachometer. The 


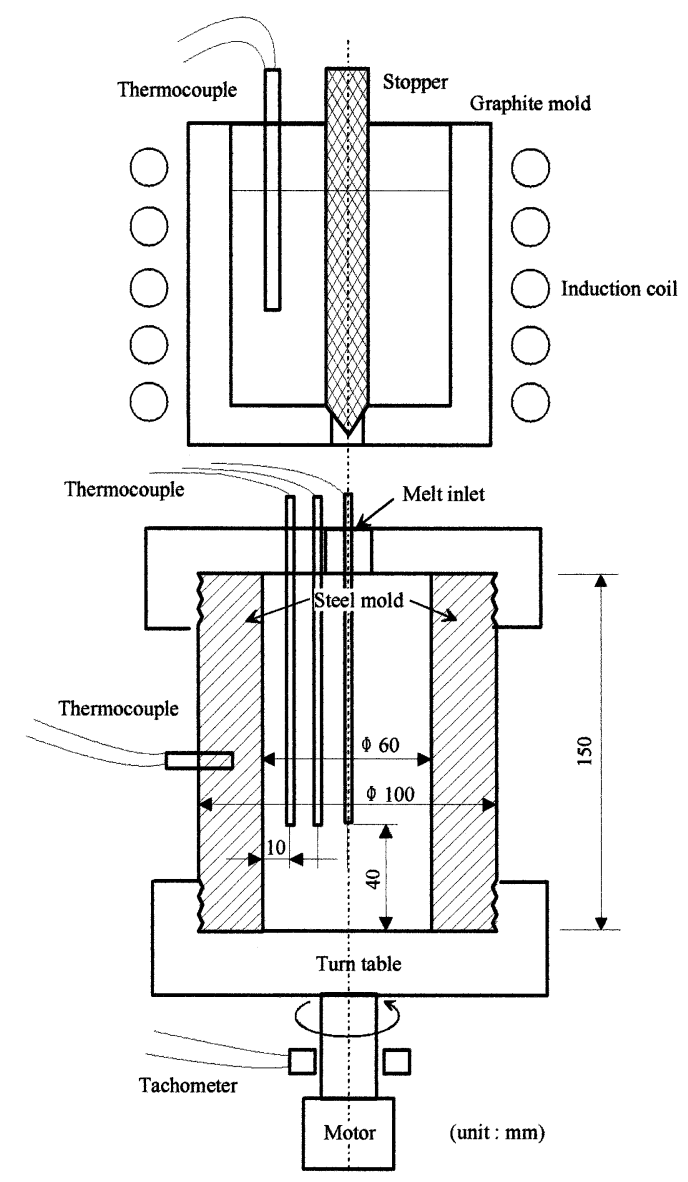

Fig. 1. Schematic diagram of a vertical centrifugal casting apparatus.

melt pouring method with the aid of a stopper within a graphite crucible could avoid the slag layer on the free surface of the melt entering into the rotating mold, and get a nearly constant flow pattern.

After solidification, the centrifugal cast ingots were sectioned horizontally at one third of the height from the bottom to avoid the appearance of the columnar grains growing from the mold bottom surface. After mechanically polished, the specimens of $\mathrm{Al}-\mathrm{Si}$ and $\mathrm{Al}-\mathrm{Cu}$ alloys were etched with Keller solution $\left(75 \mathrm{ml} \mathrm{HCl}+25 \mathrm{ml} \mathrm{HNO}_{3}+5 \mathrm{ml}\right.$ $\mathrm{HF})$ and Tucker solution $\left(92 \mathrm{~m} l \mathrm{H}_{2} \mathrm{O}+6 \mathrm{~m} l \mathrm{HNO}_{3}+2 \mathrm{ml}\right.$ $\mathrm{HF})$, respectively.

\section{Model Description and Numerical Method}

\subsection{Nucleation and Growth Kinetics}

In the present study, the continuous nucleation model was adopted in which two different Gaussian distributions were considered for describing the heterogeneous nucleation both on the mold wall and in the bulk liquid. The increase of grain density $d n$ is induced by an increase in the undercooling $d(\Delta T)$ according to the following Gaussian distribution: ${ }^{15)}$

$$
\frac{d n}{d(\Delta T)}=\frac{n_{\max }}{\sqrt{2 \pi} \Delta T_{\sigma}} \exp \left[-\frac{1}{2}\left(\frac{\Delta T-\Delta T_{\mathrm{mn}}}{\Delta T_{\sigma}}\right)^{2}\right] \ldots \ldots
$$

where $\Delta T_{\mathrm{mn}}$ is the mean nucleation undercooling, $\Delta T_{\sigma}$ is the standard deviation, and $n_{\max }$ is the maximum density of nuclei given by the integral of this distribution from 0 to $\infty$. Thus, the density of grains $n(\Delta T)$ formed at any undercooling $\Delta T$, is given by

$$
n(\Delta T)=\int_{0}^{\Delta T} \frac{d n}{d(\Delta T)} d \Delta T
$$

The banding phenomenon in vertical centrifugal castings is accompanied by the distinctive structural discontinuity due to the existence of centrifugal force and fluid flow. Since the present model was not coupled with convection and assumed to operate without the bulk liquid flow, any nuclei or thin dendrites that were to be formed would stay in the same position. In order to compensate for the effects of centrifugal force and fluid flow on the bulk nucleation distribution in the different region of the casting, the bulk nucleation rule should be modified according to the nucleation mechanism under the centrifugal casting conditions.

Several theories have been proposed to explain the origin of the nuclei for forming the exquiaxed zone in the unrefined alloys, such as the constitutional undercooling theory, ${ }^{16)}$ the free chill crystal theory, ${ }^{17)}$ the dendrite arm remelting theory, ${ }^{18)}$ the showering down theory, ${ }^{19)}$ and the dendrite fragmentation or termed grain multiplication theory. ${ }^{20)}$ It is firstly necessary to determine if all or only some of the proposed mechanisms are active and which ones are mainly responsible for the occurrence of the special structure features formed in the centrifugal casting. In conventional centrifugal casting, there must exist two stages: the violent turbulent flow stage in the teeming period and the stable flow stage in which the motion of the melt synchronizes with the rotating mold. Upon pouring the liquid into a rotating mold, a lot of crystals nucleate on the cold mold wall as a result of large thermal undercooling. These chill crystals grow out from the wall with a necked shape, owing to the solute segregation and subsequently detached through mechanical breakage or thermal fluctuations in the liquid. The effects of the violent convection and turbulence due to the melt pouring into the rotating mold enhance the crystal detachment and transport them into the bulk liquid. The deflection of columnar grains is frequently observed in the centrifugal casting. It is mostly due to the severe chill effect from the mold conditions and the relative movement between the liquid metal and the solidified metal. ${ }^{13,21,22)}$ Therefore, it is clear that the well-oriented dendritic columnar grains growing from the chill zone are also influenced by turbulent flow due to the relative movement between the liquid and the mold, broken or remelted into fine fragments, and dispersed into the flowing melt. It was assumed that the dominant nucleation mechanisms are the free-chill crystal mechanism as well as the dendrite fragmentation mechanism. All crystals formed in the initial stage of centrifugal casting experience a strong centrifugal field and have a tendency to float out radially. Accordingly, the motion of nuclei is affected by the turbulent flow and the centrifugal force in the initial stage. The former effect is basically proportional to the surface area of each crystal and the latter effect to the volume of each crystal. For larger crystals, which have lower area-to-volume ratios, the centrifugal force dominates and drives them to move outwards. 
Table 1. Growth kinetics used in the present calculation.

\begin{tabular}{|c|c|}
\hline Alloy system & $\begin{array}{c}\text { Growth velocity }(\mathrm{m} / \mathrm{sec}) \\
(\Delta T)=\kappa_{1} \cdot \Delta T^{2}+\kappa_{2} \cdot \Delta T^{3}\end{array}$ \\
\hline \multirow{2}{*}{$\mathrm{Al-Cu}$ alloy $(<8.0 \mathrm{mass} \% \mathrm{Cu})$} & $\mathrm{k}_{1}=1.16633 \times 10^{-4} \cdot(\mathrm{mass} \% \mathrm{Cu})^{-1.24319}$ \\
& $\mathrm{k}_{2}=5.39996 \times 10^{-4} \cdot(\mathrm{mass} \% \mathrm{Cu})^{-2.13518}$ \\
\hline & $\mathrm{k}_{1}=1.2045 \times 10^{-6}-0.00146 \cdot \exp (-(\mathrm{mass} \% \mathrm{Si}+1.31556) / 1.23852)$ \\
& $-0.78671 \cdot \exp (-(\mathrm{mass} \% \mathrm{Si}+1.31556) / 0.37001)$ \\
& $\mathrm{k}_{2}=0.00031 \times(\operatorname{mass} \% \mathrm{Si})^{-2.603}$ \\
\hline
\end{tabular}

Conversely, the turbulence force for smaller crystals dominates and causes them to scatter far from the mold wall. ${ }^{1)}$ Moreover, the crystals that located near the center could be expected to become further smaller and even disappear because of the remelting effect by the higher melt superheat in the center region. It is well known that the equilibrium liquidus of a crystal will decrease with the decrease of crystal size. This means that the bulk nucleation undercooling $\Delta T_{b}$, which is needed for a crystal becoming an active nucleus for growth, would increase as the crystal size decreases. Therefore, in the centrifugal casting, the mold rotation will result in that firstly the nucleation in the region far from the center increases, and secondly they could become active under a relative lower undercooling than that of the nuclei located near the center. The equiaxed grains survive as a result of the constitutional undercooling ahead of the columnar interface. Based on the mechanism proposed above, the modified bulk nuclei generation conditions were thus implemented: the nucleation density decreases, whereas the nucleation undercooling increases along the direction from the mold surface to the final solidification zone.

For the sake of simplicity, the surface nucleation undercooling was assumed to be a constant value of $0.5^{\circ} \mathrm{C} .{ }^{23)}$ The standard deviation $\Delta T_{\sigma}$ in Eq. (1) was chosen to be $0.1^{\circ} \mathrm{C}$, so that all the potential nucleation sites would become active almost at one critical undercooling. The growth velocity of a dendrite tip at a certain undercooling and concentration in the melt was calculated using the KGT (KurzGiovanol-Trivedi) model. ${ }^{24)}$ The parameters used to evaluate the relationships between the growth velocity and local undercooling for $\mathrm{Al}-\mathrm{Si}$ and $\mathrm{Al}-\mathrm{Cu}$ alloys are given in Table 1.

Nuclei formed either on the mold wall or in the bulk liquid were assumed to have a random crystallographic orientation within 48 classes. The preferential growth direction corresponds to $\langle 10\rangle$ for cubic metals.

\subsection{Classical Cellular Automaton}

This work adopted a classical cellular automaton (CA) technique ${ }^{14)}$ to simulate the macrostructure in vertical centrifugal casting. The classical CA model for nucleation and growth simulation consists of (1) the geometry of the cells, (2) the state of the cells, (3) the neighborhood configuration, and (4) several transition rules that determine the state of a given cell during one time step. In this work, the calculation domain is divided into the uniform square arrangement of cells. Each cell is characterized by different variables (such as temperature, crystallographic orientation) and state (solid or liquid). The selected neighborhood configuration contains eight neighbors in the first layer configuration.
The cellular automaton evolves in discrete time step, and the state of a cell at a particular time is calculated from the local rule, such as the nucleation and growth kinetics. At the beginning of simulation, the nucleation sites both on the mold wall and in the bulk liquid were randomly set according to Eq. (1). The nucleation sites on the mold wall or in the bulk liquid were identified with a reference integer that related to a corresponding undercooling for nucleation. During one iteration loop of calculation, the change of a cell state from "liquid" to "solid" is initiated either by nucleation or by the growth of a solid cell. If a given liquid cell is a predetermined nucleation site and the local undercooling is larger than that which is necessary for nucleation, this cell becomes to a solid and its corresponding index is randomly defined to be an integer among 48 classes, which represents its crystallographic orientation.

Once a cell has nucleated, it will grow with a preferential direction corresponding to its crystallographic orientation and the growth velocity is determined by the local undercooling using the KGT model. When the growth front of a solid cell touches the center of its neighboring liquid cells, the neighboring cell transforms its state from liquid to solid and gets the same orientation index as the solid cell.

\subsection{Macroscopic Thermal Transport}

Considering the geometric symmetry in the cross section of the vertical centrifugal casting, the present model adopted a one-dimensional polar coordinate to calculate the temperature profile. The convective heat transfer in the melt was not considered. The governing equation for the one-dimensional polar coordinate transient heat conduction is given by

$$
\rho C_{\mathrm{p}} \frac{\partial T}{\partial t}=\frac{1}{r} \frac{\partial}{\partial r}\left(\lambda \cdot r \frac{\partial T}{\partial r}\right)+\rho L \frac{\partial f_{\mathrm{s}}}{\partial t}
$$

where $T$ is temperature, $\sigma$ is the density, $C_{\mathrm{p}}$ is the specific heat, is the thermal conductivity, $L$ is the latent heat of freezing, and $f_{\mathrm{s}}$ is the solid fraction, respectively.

The boundary conditions at the casting/mold interface and the mold/air interface are given by

At the casting/mold:

$$
q=h_{\mathrm{cm}}\left(T_{\text {mold }}-T_{\text {casting }}\right)
$$

At the mold/air:

$$
q=h_{\text {ma }}\left(T_{\text {air }}-T_{\text {mold }}\right)
$$

where is the heat fiux, $T_{\text {casting }}, T_{\text {mold }}$, and $T_{\text {air }}$ are the temperatures of the casting, the mold and the air at the mold sur- 


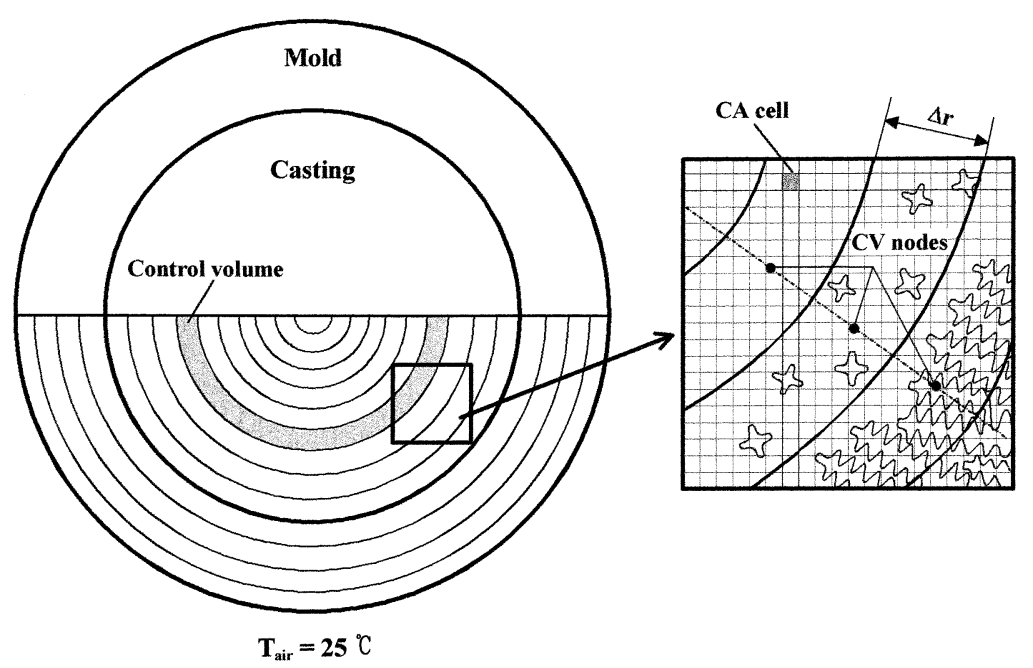

Fig. 2. Schematic diagram of superimposing CA cells with CV nodes.

faces, and $h_{\mathrm{cm}}$ and $h_{\mathrm{ma}}$ are the interfacial heat transfer coefficients at the casting/mold and the mold/air interface, respectively.

It is apparent that the contact force between the melt and the mold will increase, and the air gap between them will decrease due to the existence of centrifugal force. This will then result in the proportional increase of the interfacial heat transfer coefficient. Thus, the interfacial heat transfer coefficients $h_{\mathrm{cm}}$ with various mold rotation speeds of 0,50 , 100,200 and $400 \mathrm{rpm}$ were assumed to be 1000,1200 , 1400,1800 and $2600 \mathrm{~W} / \mathrm{m}^{2} \mathrm{~K}$, respectively. The interfacial heat transfer coefficient at the mold/air interface $h_{\text {ma }}$ was considered to be $50 \mathrm{~W} / \mathrm{m}^{2} \mathrm{~K}$. The governing equations and the boundary conditions shown in Eqs. (3) to (5) were solved by the explicit finite difference algorithm.

\subsection{Coupling of the Cellular Automaton and the Finite Volume Method}

The present macrostructure simulation model consists of two schemes: the cellular automaton $(\mathrm{CA})$ to simulate the evolution of solidification structures and the finite volume method (FVM) in the one-dimensional polar coordinate to calculate the heat transfer in casting.

Figure 2 indicates the schematic drawing of the calculation domain with control volumes $(\mathrm{CV})$ coupled with $\mathrm{CA}$ cells. Considering the geometric symmetry, a half area was taken for the calculation domain to save the calculation time. As shown in Fig. 2, the whole domain including the mold and the casting was divided into 100 semicircle annulus control volumes with a uniform thickness of $\Delta r$ $(\Delta r=0.5 \mathrm{~mm})$ for heat transfer calculation, and the $\mathrm{CV}$ within the casting domain was further divided into the uniform square CA cells for structure calculation. The total cell number was 141680 and the cell size was taken to be $100 \mu \mathrm{m}$. For the sake of simplicity and the reduction of calculation time, the inner node $\mathrm{CV}$ scheme was adopted and the temperatures of all cells within a $\mathrm{CV}$ were assumed to be uniform. Based on the calculated temperature profile in the cells, the calculation of nucleation and growth was carried out by the classical CA model.

In order to reduce the calculation time, two kinds of time step were used one for $\mathrm{CV}$ temperature and the other for
CA structure calculation, respectively. These are given by CV time step:

$$
\delta t_{\mathrm{T}}=\frac{\Delta r_{\mathrm{CV}}^{2} \times \rho C_{\mathrm{P}}}{3 \times \lambda}
$$

where $\Delta r_{\mathrm{CV}}$ is the $\mathrm{CV}$ thickness of the semicircle annulus, $\rho C_{\mathrm{p}}$ is the volumetric specific heat and $\lambda$ is the thermal conductivity.

CA time step:

$$
\delta t_{\mathrm{CA}}=\frac{1}{4.5} \times \frac{\Delta x}{V_{\max }}
$$

where $\Delta x$ is the cell size and $V_{\max }$ is the maximum growth velocity obtained by scanning the growth velocities of all interface liquid cells during each time step.

When a liquid cell solidifies by nucleation or growth, it will release the latent heat, which is given by

$$
\Delta T_{\mathrm{L}}=\frac{\Delta H_{\mathrm{V}} \times \Delta x^{2}}{\rho C_{\mathrm{p}} \times \Delta S_{\mathrm{CV}}^{\mathrm{i}}}
$$

where $\Delta T_{L}$ is the temperature recovered by one solidified cell, $\Delta H_{\mathrm{V}}$ is the volumetric latent heat and $\Delta S_{\mathrm{CV}}^{\mathrm{i}}$ is the area of the CV including the newly solidified cell. The temperature of the CV node is then recovered by the amount of latent heat released by the total newly solidified cells included in the CV. Using these updated temperatures, macroscopic heat transfer calculation can be continued. This series of calculation will be repeated until the end of solidification. The flowchart of the present calculation is shown in Fig. 3.

\subsection{Modified Cellular Automaton}

A modified Cellular Automaton (MCA) model has been recently developed to predict the evolution of dendritic microstructures. ${ }^{14)}$ The MCA model accounts for the solute partition between liquid and solid, and solute diffusion in these two phases. It is assumed that the solid and liquid compositions at the solid/liquid interface are in equilibri- 


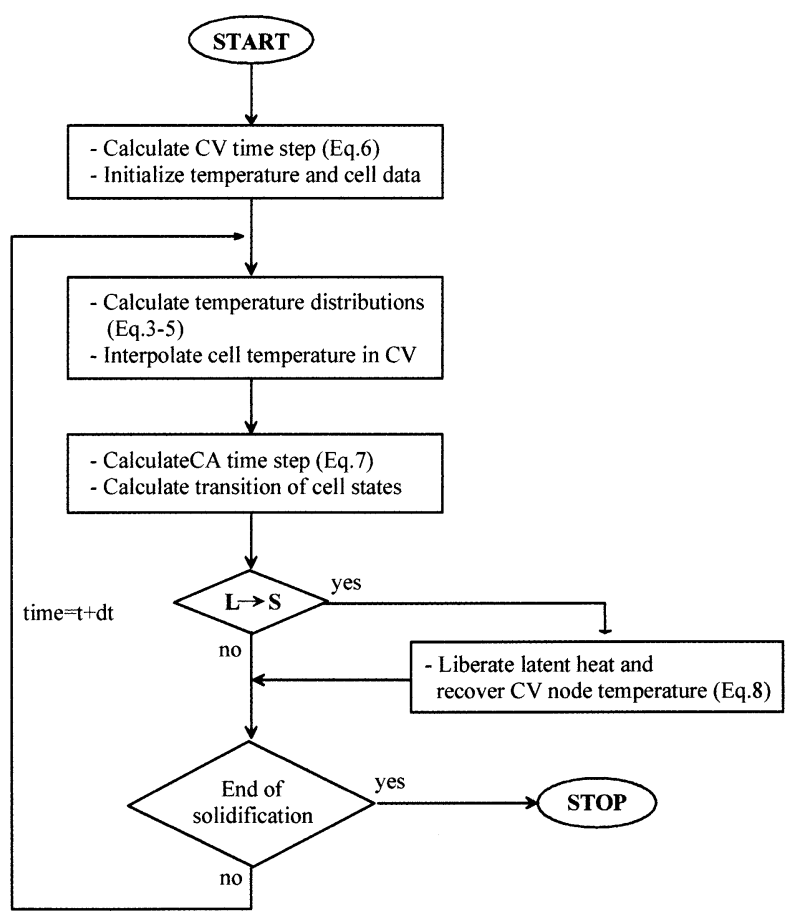

Fig. 3. Flowchart of the CA-CV coupling and the macrostructure calculation procedure.

um.

$$
C_{\mathrm{s}}^{*}=k C_{1}^{*}
$$

where $k$ is the partition coefficient, $C_{\mathrm{s}}^{*}$ and $C_{1}^{*}$ are the interface equilibrium concentration in the solid and liquid phases, respectively.

As the solidification proceeds, the solidified cell rejects solute to its neighbor liquid cells. Diffusion within the entire domain is then simulated. The governing equation for the solute redistribution in the liquid region is given by

$$
\frac{\partial C_{1}}{\partial t}=\frac{\partial}{\partial x}\left[D_{1} \frac{\partial C_{1}}{\partial x}\right]+\frac{\partial}{\partial y}\left[D_{1} \frac{\partial C_{1}}{\partial y}\right]+C_{1}(1-k) \frac{\partial f_{\mathrm{s}}}{\partial t}
$$

where $t$ is time, $D_{1}$ is the solute diffusion coefficient in the liquid phase, $f_{\mathrm{s}}$ is the solid fraction, $k$ is the partition coefficient, and $x$ and $y$ are the domain coordinates, respectively. The last term on the right hand side of the Eq. (10) indicates the amount of solute rejected at the solid/liquid interface.

The governing equation for diffusion in solid is given by

$$
\frac{\partial C_{\mathrm{s}}}{\partial t}=\frac{\partial}{\partial x}\left[D_{\mathrm{s}} \frac{\partial C_{\mathrm{s}}}{\partial x}\right]+\frac{\partial}{\partial y}\left[D_{\mathrm{s}} \frac{\partial C_{\mathrm{s}}}{\partial y}\right]
$$

where $D_{\mathrm{s}}$ is the diffusion coefficient of solute in solid. The zero-flux boundary conditions were used for the cells located at the surface of the calculation domain. An explicit finite difference scheme was applied to calculate the solute diffusion in both the solid and liquid phases. The thermal and physical properties used in the present calculation are listed in Table 2. In addition, in the MCA model, the constitutional undercooling effect is incorporated on the equi-

\begin{tabular}{|c|c|c|c|}
\hline \multicolumn{2}{|r|}{ Designation } & \multirow{2}{*}{$\begin{array}{c}\text { Value } \\
933.0 \\
\end{array}$} & \multirow{2}{*}{\begin{tabular}{|l|} 
Remark \\
Al-Cu alloy \\
\end{tabular}} \\
\hline $\mathrm{T}_{\mathrm{m}}$ & Melting temperature $(\mathrm{K})$ & & \\
\hline \multirow{2}{*}{$\mathrm{T}_{\text {eut }}$} & \multirow{2}{*}{ Eutectic temperature $(\mathrm{K})$} & 822.0 & Al-Cu alloy \\
\hline & & 850.0 & Al-Si alloy \\
\hline \multirow{2}{*}{$\mathrm{C}_{\text {eut }}$} & \multirow{2}{*}{ Eutectic concentration (mass\%) } & 33.3 & Al-Cu alloy \\
\hline & & 10.77 & Al-Si alloy \\
\hline \multirow{2}{*}{$\mathrm{k}$} & \multirow{2}{*}{ Partition coefficient } & 0.17 & Al-Cu alloy \\
\hline & & 0.117 & Al-Si alloy \\
\hline \multirow{2}{*}{$\mathrm{m}$} & \multirow{2}{*}{ Slope of the liquidus line (K/mass\%) } & -3.36 & Al-Cu alloy \\
\hline & & -6 & Al-Si alloy \\
\hline \multirow{2}{*}{$\mathrm{C}_{\mathrm{p}}$} & \multirow{2}{*}{ Specific heat $(\mathrm{J} / \mathrm{kg} \cdot \mathrm{K})$} & 1086 & Al alloy (casting) \\
\hline & & 580 & SKD61 (mold) \\
\hline \multirow{2}{*}{$\rho$} & \multirow{2}{*}{ Density $\left(\mathrm{kg} / \mathrm{m}^{3}\right)$} & 2780 & Al alloy (casting) \\
\hline & & 7890 & SKD61 (mold) \\
\hline \multirow{2}{*}{$\lambda$} & \multirow{2}{*}{ Thermal conductivity $(\mathrm{W} / \mathrm{m} \cdot \mathrm{K})$} & 192.5 & Al alloy (casting) \\
\hline & & 14.4 & SKD61 (mold) \\
\hline$\Delta \mathrm{H}_{\mathrm{v}}$ & Volumetric latent heat $\left(\mathrm{J} / \mathrm{m}^{3}\right)$ & $1.107 \cdot 10^{9}$ & Al alloy (casting) \\
\hline $\mathrm{D}_{\mathrm{L}}$ & Solute diffusion coefficient in liquid $\left(\mathrm{m}^{2} / \mathrm{sec}\right)$ & $1.0 \cdot 10^{-9}$ & Al alloy (casting) \\
\hline $\mathrm{D}_{\mathrm{S}}$ & Solute diffusion coefficient in solid $\left(\mathrm{m}^{2} / \mathrm{sec}\right)$ & $1.0 \cdot 10^{-12}$ & Al alloy (casting) \\
\hline
\end{tabular}

Table 2. Thermal and physical properties used in the calculation.

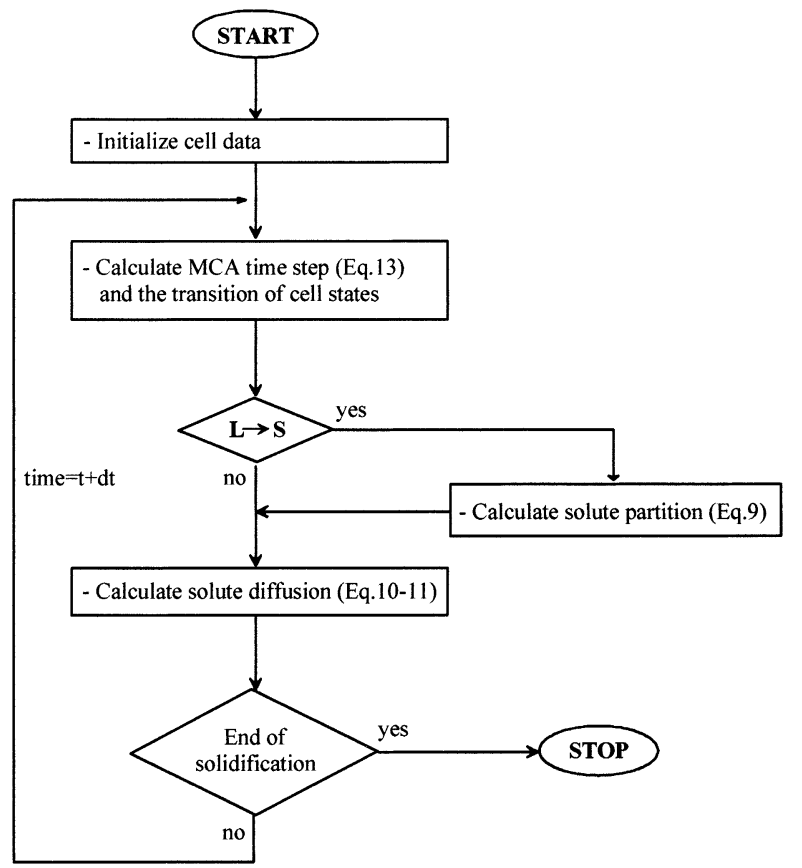

Fig. 4. Flowchart of the microstructure calculation procedure by a modified CA model.

librium interface temperature. Therefore, the local undercoling $\Delta T\left(t_{\mathrm{n}}\right)$ at time $t_{\mathrm{n}}$ is given by

$$
\Delta T\left(t_{\mathrm{n}}\right)=T_{1}+\left[C\left(t_{\mathrm{n}}\right)-C_{0}\right] m-T\left(t_{\mathrm{n}}\right) .
$$

where $T_{1}$ is the equilibrium liquidus temperature, $m$ is the liquidus slope, $C_{0}$ is the initial concentration of the alloy, and $C\left(t_{\mathrm{n}}\right)$ and $T\left(t_{\mathrm{n}}\right)$ are the concentration and the temperature of the liquid/solid interface at time $t_{\mathrm{n}}$, respectively.

As the thermal diffusivity of metallic alloys is $3-4$ orders of magnitude greater than the solute diffusivity, the kinetics for structural evolution can be assumed to be solute diffusion-controlled, and thus the thermal diffusion can be neglected at the microscopic scale. ${ }^{25}$ ) The temperature field in the domain of microstructure calculation by the MCA model was therefore assumed to be uniformly cooled, which was obtained by the macroscopic temperature calculation (see the Sec. 3.3). Figure 4 shows the flowchart of 
the microstructure calculation by the MCA model. The MCA calculation time step is given by

$$
\delta t_{\mathrm{MCA}}=\frac{1}{4.5} \min \left[\frac{d x}{V_{\max }}, \frac{\Delta x^{2}}{D_{1}}\right] \ldots \ldots \ldots \ldots \ldots
$$

where $V_{\max }$ is the maximum growth velocity obtained by scanning the growth velocities of all interface liquid cells during each time step and $D_{1}$ is the solute diffusion coefficient in liquid phase.

\section{Results and Discussion}

The present model was applied to simulate the solidification grain structures of $\mathrm{Al}-\mathrm{Si}$ and $\mathrm{Al}-\mathrm{Cu}$ alloys in vertical centrifugal casting.

\subsection{Effects of Mold Rotation Velocity}

Figure 5 shows the simulated and experimental macrostructures of an $\mathrm{Al}-1.0 \mathrm{mass} \% \mathrm{Si}$ alloy solidified centrifugally with the various mold velocities of (a) $0 \mathrm{rpm}$, (b) $50 \mathrm{rpm}$, (c) $100 \mathrm{rpm}$, (d) $200 \mathrm{rpm}$, and (e) $400 \mathrm{rpm}$. The mold rotation speeds relate to the tangential velocities on the mold surface as $0,150,300,600$, and $1200 \mathrm{~mm} / \mathrm{sec}$, respectively. The figures in the left column indicate the simulated grain structures and the right ones the experimental results. Figure 5(a) exhibits a typical conventional casting macrostructure in which three distinct zones could be observed: the chill zone near the mold surface, the columnar zone growing from the chill zone, and finally the equiaxed zone in the center. Comparing Fig. 5(b) with Fig. 5(a), it could be noted that the equiaxed grain size in the center region of Fig. 5(b) is a little larger than that of Fig. 5(a). Besides, the columnar zone is a little narrower in the Fig. 5(b) than that in the Fig. 5(a). These are due to the fact that the decrease of the mold rotation velocity results in a little change of the bulk nucleation distribution in liquid. When the mold rotation speed increases to $100 \mathrm{rpm}$, the obvious equiaxed-tocolumnar transition occurs as shown in Fig. 5(c). It could be seen from Figs. 5(c) through 5(e) that the higher the mold rotating speed was, the broader the second columnar zone developed. This trend shows that the equiaxed zone is not developed in the center region because there is no pile up of solute ahead of the primary columnar grains due to turbulent flow, and therefore no constitutional undercooling for the formation of the equiaxed zone. Thus, the equiaxed grains are formed in the initial solidification stage and affected by the centrifugal force. It also could be observed from these pictures that as the mold rotation speed increases, the equiaxed grain size becomes finer, and the primary columnar zone becomes narrower. In addition, the inclination of the primary columnar grains to the same direction of the mold rotation is observed. This means that a considerable fluid flow exists along the circumferential direction, since the columnar dendrites growing in a flowing melt tend to deflect towards the upstream direction. ${ }^{13)}$ It is considered to be due to the fact that as the mold rotation speed increases, the initial convention and turbulence in the liquid become more intensified, enhancing the detachment of chill crystals and arms of the primary dendritic columnar grains.
Moreover, during the floating period of detached crystals in the melt, they probably collide with each other and fracture into small. These fragments, in turn, act as new nuclei. The effects of increasing the mold rotation speed result in the increase of the bulk nucleation density and the finer equiaxed grain size.

In addition, the centrifugal force is known as to depend on the mold rotation velocity, which is given by

$$
f=m \cdot r \cdot \omega^{2} \text {...... }
$$

where $f$ is the centrifugal force, $m$ is the nucleus mass, $r$ is the distance between the nucleus position and the center, and $\omega$ is the rotation velocity. Accordingly, the centrifugal force would rapidly increase as the mold rotation velocity increases, which accelerates the bulk nuclei moving toward and pressurizing against the mold wall, and results in the thin primary columnar zone and the broad secondary columnar zone.

\subsection{Effects of Solute Content}

In order to investigate the effects of alloy composition on macrostructures in vertical centrifugal casting, both simulation and experiment were carried out on four different alloy compositions with a fixed mold rotation velocity of 200 rpm. Figure 6 indicates the obtained macrostructures: (a) $0.0 \mathrm{mass} \% \mathrm{Si}$, (b) $0.5 \mathrm{mass} \% \mathrm{Si}$, (c) $1.0 \mathrm{mass} \% \mathrm{Si}$, and (d) $2.0 \mathrm{mass} \% \mathrm{Si}$. The pictures in the left column indicate the predicated grain structures and the ones in the right the experimental results. As shown in Fig. 6(a), there is no equiaxed zone in the centrifugal casting ingot of a high purity aluminum ( $>99.99$ mass $\%)$. According to the free chill crystal theory, when pouring the high purity melt into the mold, the chill crystals formed at the cold wall could not grow out with the necked shape because of no solute segregation. It is thus very difficult for these chill crystals to be detached and transported by the convection and turbulence, resulting in few bulk nuclei in the melt. Therefore, the dendrites originated from the surface nuclei with a favorable orientation can grow in the direction roughly perpendicular to the mold wall and develop up to the center of the ingot, which leads to the fully columnar grain structure.

As shown in Figs. 6(b) through 6(d), the equiaxed and secondary columnar zones were formed in the concentrated region. As the concentration increases, the length of the primary columnar zone becomes narrower and that of the equiaxed zone becomes wider, and the grain size in the equiaxed zone becomes finer. These phenomena could be easily understood by the nucleation mechanisms and dendrite growth kinetics. As the concentration increases, the chill wall crystals would be more easily detached due to the increase of the solute segregation effect, which results in the increase of the bulk nucleation density. Furthermore, for the less concentrated alloys, the dendrite growth velocity is faster even under the same undercooling and the occurrence of heterogeneous nucleation in the bulk liquid becomes more difficult. Figure $7^{26)}$ presents the variation in number of grains per unit volume as a function of undercooling for two kinds of solute content $C_{1}$ and $C_{2}\left(C_{2}>C_{1}\right)$. It is very clear that under any conditions, the nucleation density for higher concentration is larger than that for lower concentration. 

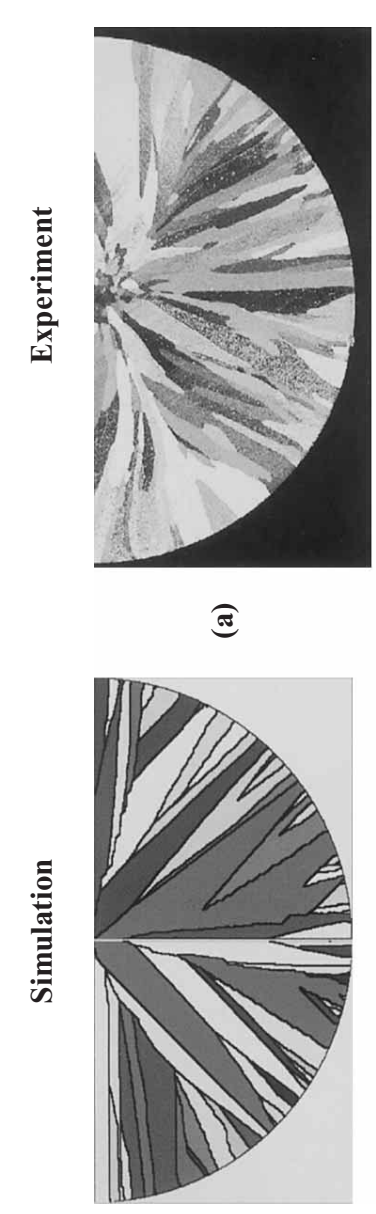

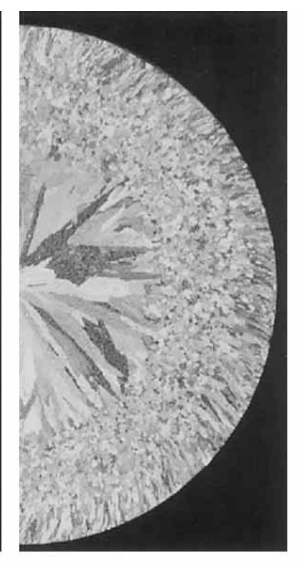

$\widehat{\varrho}$
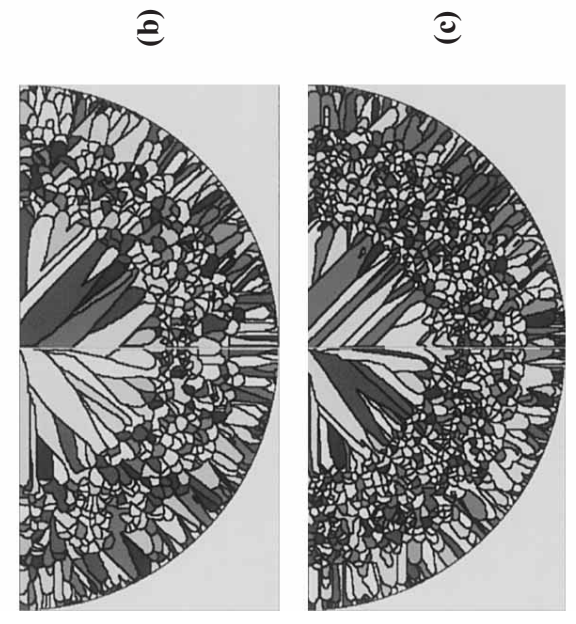

อ
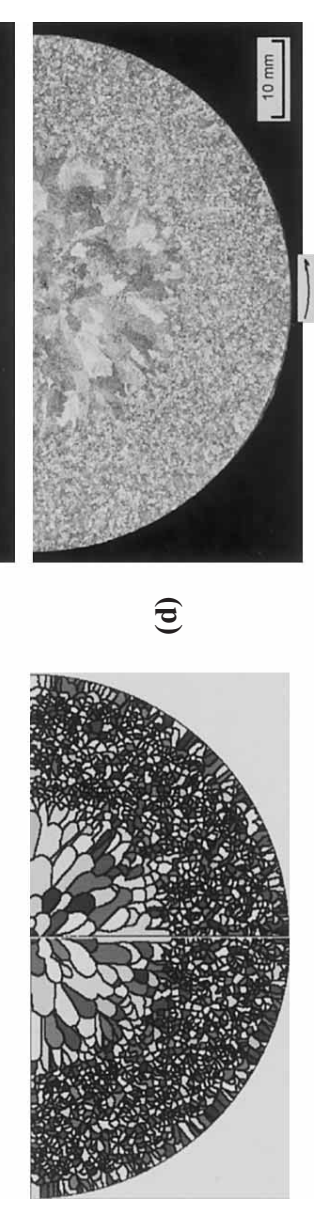

잉
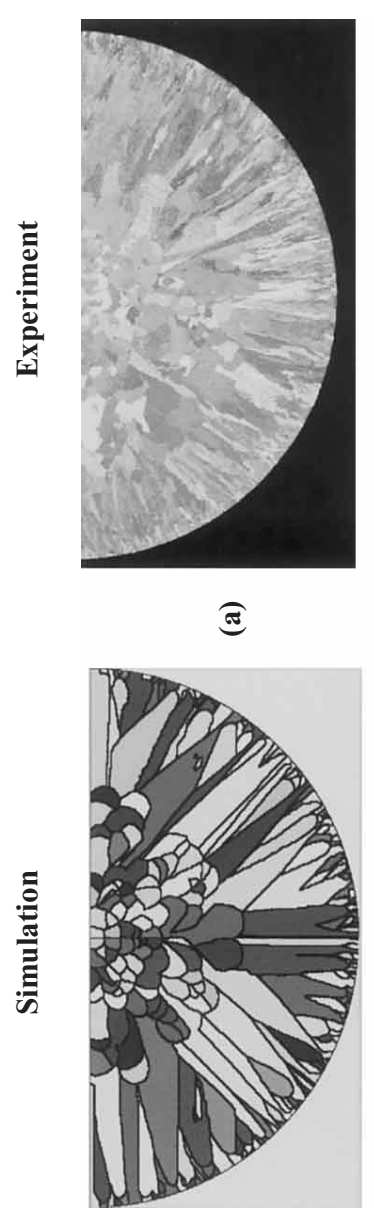

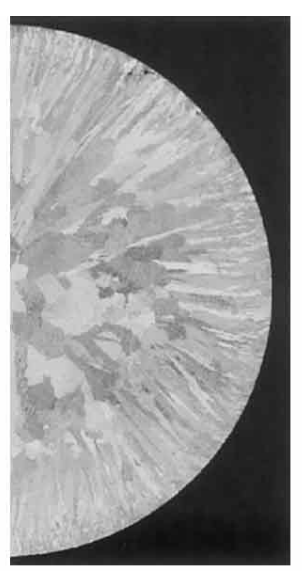

$\widehat{\varepsilon}$

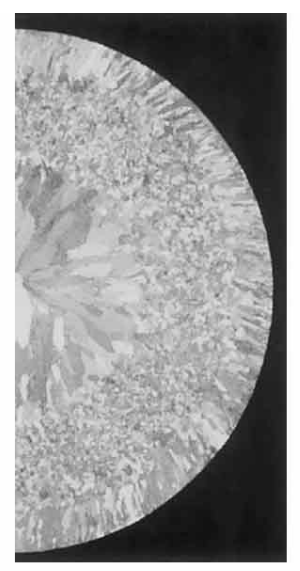

(9)
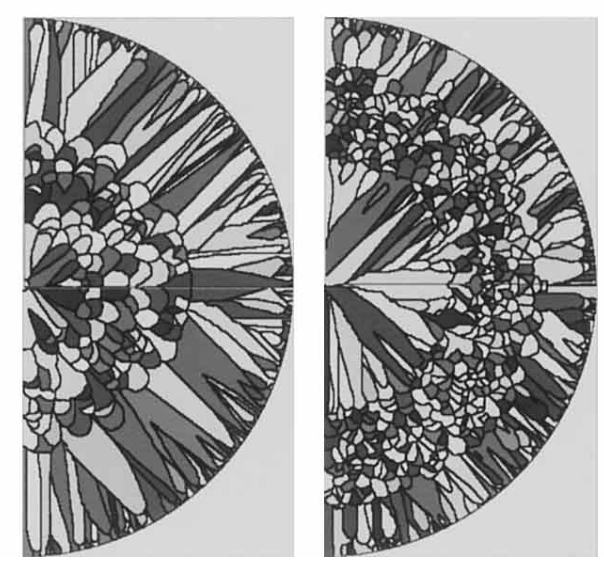

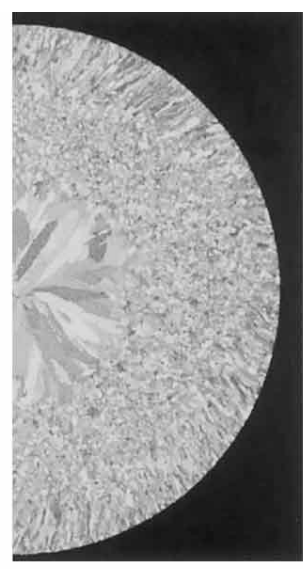

ङ

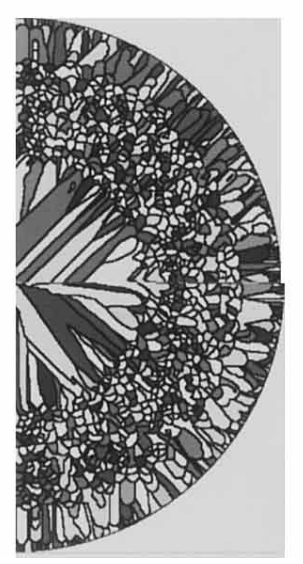

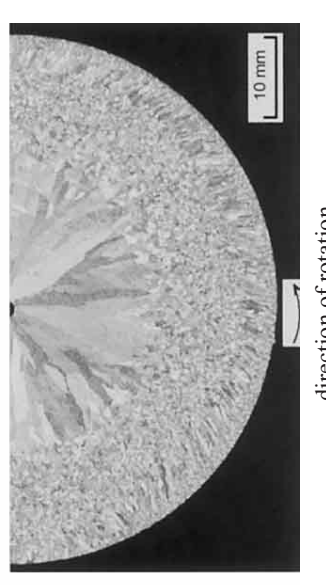

త

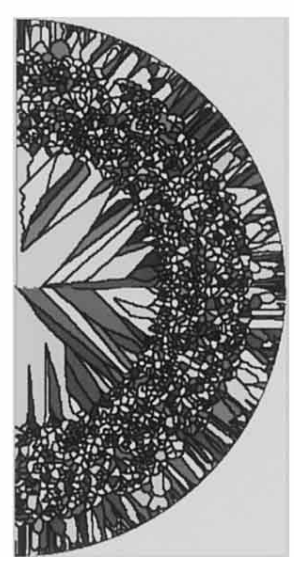

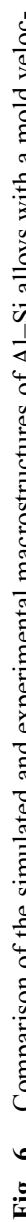

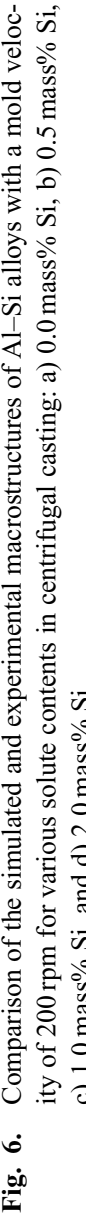

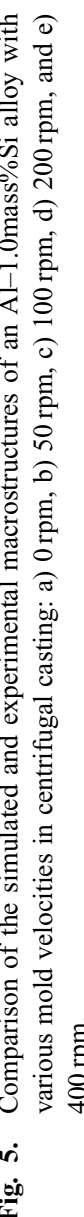


It is also worth to note that in case of solute concentration up to 2.0 mass $\% \mathrm{Si}$, the secondary columnar zone is replaced by the coarse exquiaxed grain structure as shown in Fig. 6(d). This indicates that the dendrite arm remelting or breaking mechanism prevails. As the concentration increases over a certain amount, higher order arms of dendrites can easily form from a necked shape, as a result of the buildup

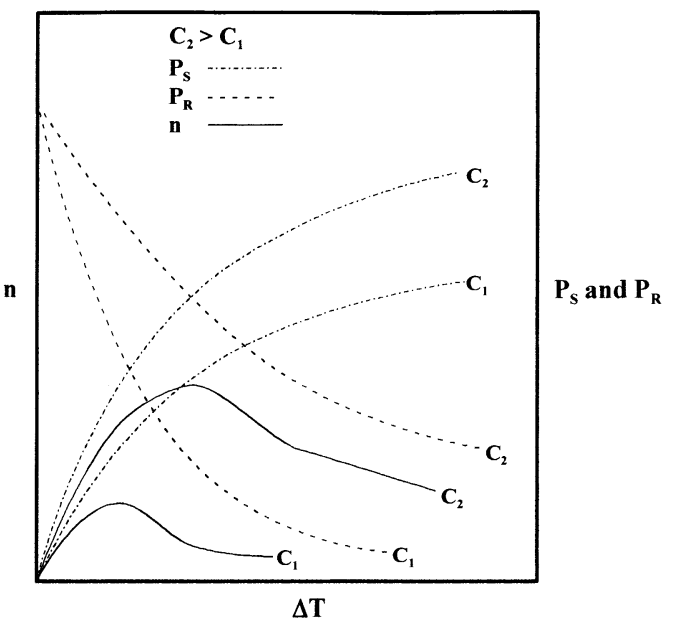

Fig. 7. Schematic illustration of the variation in number of grains per unit volume, $n$, as a function of $\Delta T$ due to two different concentrations for a unstirred melt $\left(C_{2}>C_{1}, P_{\mathrm{S}}\right.$ and $P_{\mathrm{R}}$ are the probabilities of segment formation and segment rotation, respectively). ${ }^{26)}$

Simulation

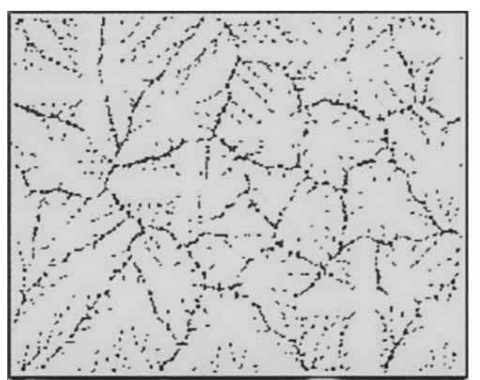

(a)

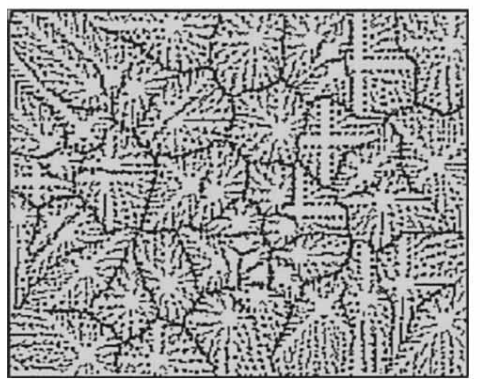

(b)

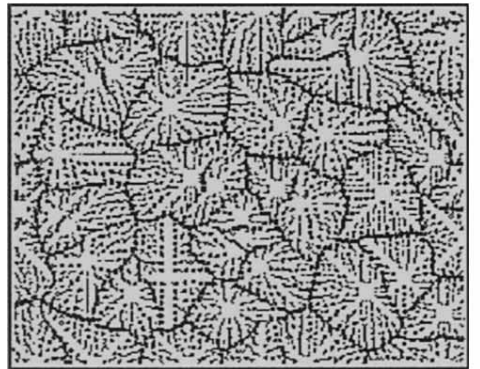

of solute in the pockets between the dendrite arms. These necked arms are more likely to be detached as the results of the latent heat of remelting or coarsening, so-called the surface energy of remelting.

In order to further investigate the effect of concentration on the microstructure, the exquiaxed dendrite morphology of $\mathrm{Al}-\mathrm{Cu}$ alloys with various solute contents were simulated by the modified cellular automaton (MCA) model. ${ }^{14}$ ) Figure 8 shows both the simulated and experimental results. The figures in the left column are the calculated microstructures, and the right column the experimented ones. Comparing Figs. 8(a) to 8(c), it is very clear that the solute content has a significant effect on the dendrite features: the higher the concentration is, the finer both the grain size and the dendrite arms are. It can be easily explained that for the higher concentrated alloys, the dendrite growth will liberate a larger amount of solute to the liquid near the solid/liquid interface, which will increase the interface instability. Therefore the side branching into the secondary arms becomes easier in the higher concentrated alloys, leading to the finer dendrite structures. Figure 8 also shows that the multi exquiaxed dendrite microstructures could be well reproduced by the MCA model.

\subsection{Effects of Mold Temperature and Pouring Tem- perature}

In general, there are two interlinked factors that influence and control the formation of grains. The first is the exis-

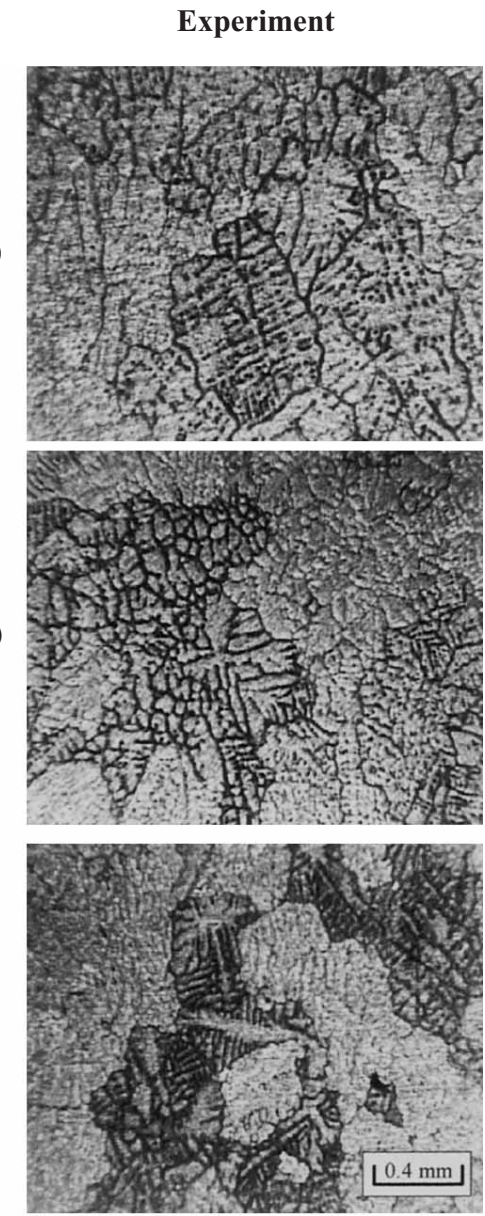

Fig. 8. Comparison of the simulated and experimental microstructures in the equiaxed zone of $\mathrm{Al}-\mathrm{Cu}$ alloys with a mold velocity of $200 \mathrm{rpm}$ for various solute contents: a) 0.5 mass $\% \mathrm{Cu}$, b) 1.0 mass $\% \mathrm{Cu}$, and c) 2.0 mass $\% \mathrm{Cu}$. 
Simulation
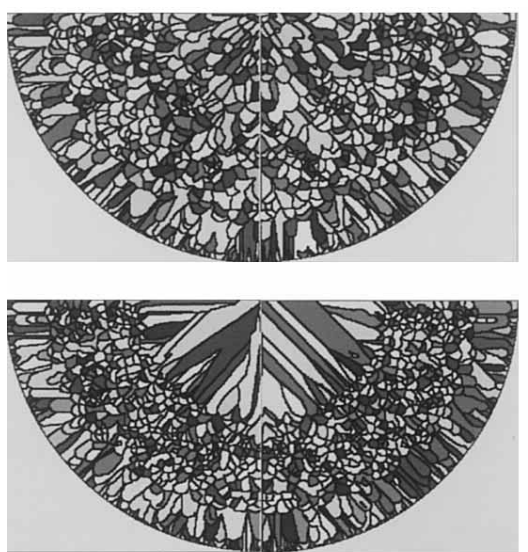

(b)

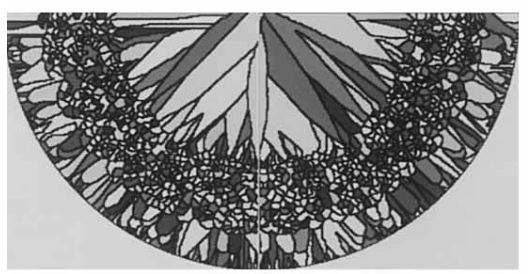

(c)
Experiment
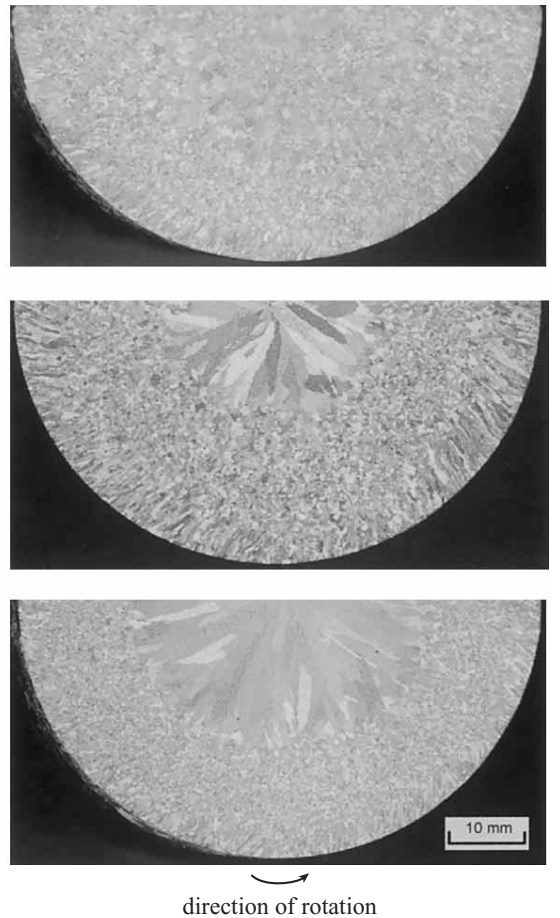

Fig. 9. Comparison of the simulated and experimental macrostructures of an $\mathrm{Al}-1.0 \mathrm{mass} \% \mathrm{Si}$ alloy with a mold velocity of $200 \mathrm{rpm}$ and a pouring temperature of $760^{\circ} \mathrm{C}$ for various mold preheat temperatures: a) $\left.10^{\circ} \mathrm{C}, \mathrm{b}\right) 70^{\circ} \mathrm{C}$, and c) $150^{\circ} \mathrm{C}$.

tence of substrates in the melt that can act as nucleation sites. And second, there has to be sufficient undercooling to facilitate the survival and growth of the nuclei. The evolution of grain structures in vertical centrifugal casting is, therefore, closely related to the mold preheating temperature and the melt superheat.

The effect of mold temperature on the macrostructure of an $\mathrm{Al}-1.0 \mathrm{mass} \% \mathrm{Si}$ alloy with a constant mold rotation speed of $200 \mathrm{rpm}$ and a pouring temperature of $760^{\circ} \mathrm{C}$ is presented in Fig. 9. The pictures in the left and right columns indicate the predicted and experimental solidification grain structures, respectively. It can be noted that with the increase of mold temperature, the length of secondary columnar zone becomes broader and the equiaxed grain size becomes finer. According to the previously reported experiments on the effects of the mold preheat on conventional solidification structures, an increase in the mold preheat temperature reduces the formation of chill wall crystals, and also reduces the likelihood of their survival as they are transported to the center of a casting. ${ }^{27)}$ Therefore, the bulk nuclei density would increases as the mold preheating temperature decreases. In addition, it is very obvious that the reduced mold preheat will cause an increase in the cooling rate within the casting. This effect will lead to an increase of the melt liquid viscosity and a decrease of the solidification time. Thus little time is available for the nuclei to move outwards in the case of low mold preheating temperature. Besides, the increase of cooling rate might cause an increase of the local undercooling in the melt, which benefits to a larger proportion of nuclei being activated, resulting in the fine equiaxed grain structure. Therefore, the low mold preheating temperature of $10^{\circ} \mathrm{C}$ exhibits a fine equiaxed grain structure, as shown in Fig. 9(a), which indi- cates that a finer grain size is produced by a higher cooling rate.

Figure 10 indicates the resultant macrostructures of an Al-1.0mass\%Si alloy centrifugally solidified with a mold rotation speed of $200 \mathrm{rpm}$ and a mold temperature of $70^{\circ} \mathrm{C}$, and with various pouring temperatures: (a) $710^{\circ} \mathrm{C}$, (b) $760^{\circ} \mathrm{C}$ and (c) $810^{\circ} \mathrm{C}$. These different pouring temperatures are corresponding to the melt superheat of $50 \mathrm{~K}, 100 \mathrm{~K}$ and $150 \mathrm{~K}$, respectively. The figures in the left column indicate the predicted structures and the ones in the right the experimental results. It can be seen from the figure that the pouring temperature of the melt has a significant effect on solidification structures. With the increase of melt superheat, the length of the second columnar zone increases, whereas the length of the primary columnar zone decreases. Besides, the grain size in the equiaxed zone becomes coarser. These trends could be easily understood in terms of an increased number of chill wall crystals or fragments formed and survived at lower levels of superheat, leading to a finer grain size. Moreover, as explained previously on the effect of the mold temperature, lower melt superheat would also cause an increase in the melt viscosity and a shorter solidification time. Therefore it is more difficult for nuclei to move outward under the low superheat condition, and this is the reason why the macrostructure with a high pouring temperature exhibits a broader secondary columnar zone and a narrower primary columnar.

\section{Conclusions}

In this study a new, coupled cellular automaton (CA) with the finite volume method has been developed for the prediction of the macrostructures formed in vertical cen- 

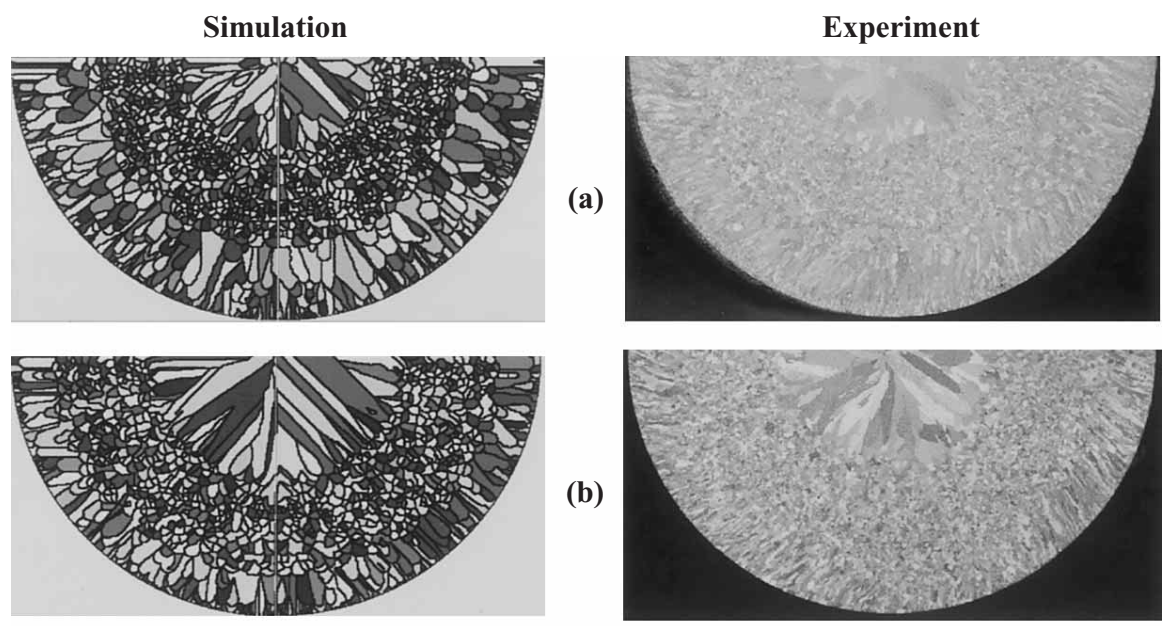

(b)
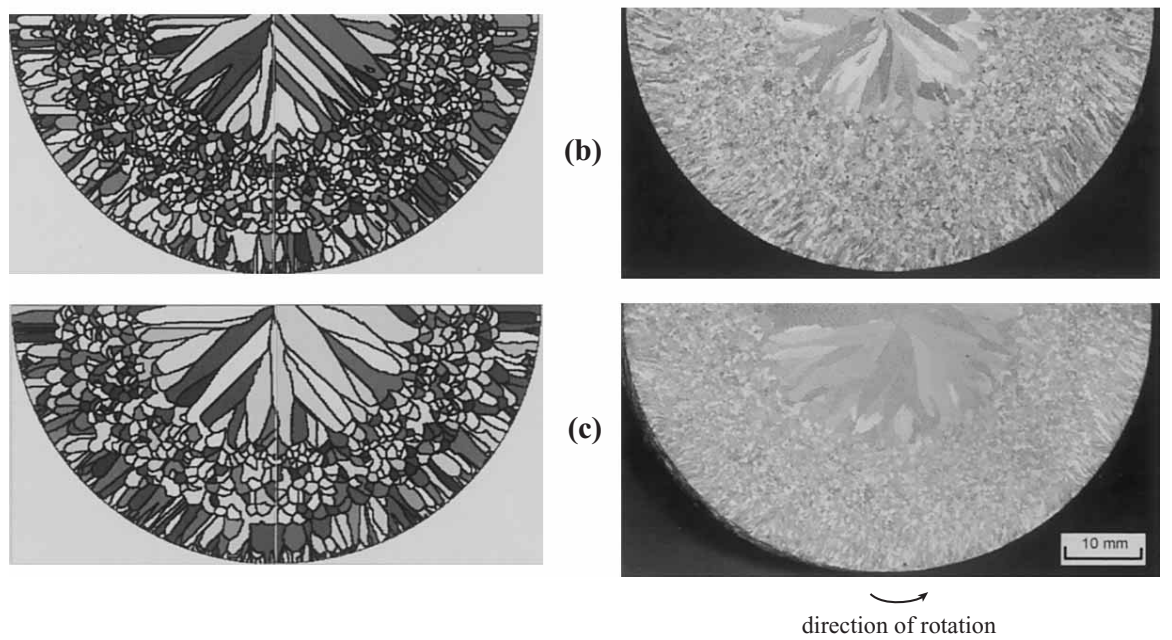

Fig. 10. Comparison of the simulated and experimental macrostructures of an $\mathrm{Al}-1.0 \mathrm{mass} \% \mathrm{Si}$ alloy with a mold velocity of $200 \mathrm{rpm}$ and a mold preheat temperature of $70^{\circ} \mathrm{C}$ for various pouring temperatures: a) $\left.710^{\circ} \mathrm{C}, \mathrm{b}\right) 760^{\circ} \mathrm{C}$, and c) $810^{\circ} \mathrm{C}$.

trifugal casting. The present model is based on a CA technique in two-dimensional right-angle coordinate for the structure simulation and the finite volume method in the one-dimensional polar coordinate for the temperature field calculation. The model can simulate the grain structures of centrifugal casting ingots with excellent computational efficiency. The microstructure simulation in the equiaxed zone with various solute contents was also carried out using a modified CA model, and the simulated results are in good agreement with those obtained by the experiment. It is ascertained that the dominant bulk nucleation mechanisms responsible for the formation of the equiaxed zone in the vertical centrifugal casting are the free-chill crystal theory and the dendrite fragmentation theory. The combination of the moderate mold rotation speed, low melt superheat, low mold preheat temperature and a slight higher solute concentration is useful to obtain the fine equiaxed grain structure in vertical centrifugal casting.

\section{Acknowledgements}

This work was supported by the Brain Korea 21 Project. The authors would like to thank Dr. M.F. Zhu, CAMP, Dr. S.M. Lee, KITECH and Dr. Y.S. Han, KIST for their helpful discussions.

\section{REFERENCES}

1) J. W. Yeh and S. H. Jong: Metall. Trans., 25A (1994), 643

2) Y. Ebisu: AFS Trans., 85 (1977), 643.

3) H. O. Howson: Proc. of The Solidification of Metals, ed. by H. Morrogh, The Iron and Steel Institute, Brighton, (1967), 334.

4) Ph. Thevoz, J. L. Desbiolles and M. Rappaz: Metall. Trans. A, 20A
(1989), 311.

5) C. Beckermann and C. Y. Wang: Modeling of Coarsening and Grain Growth, ed. by C. S. Pande and S. P. Maesh, TMS Publicatioin, Warrendale, PA, (1993), 55.

6) P. Zhu and R. W. Smith: Acta Metall., 40 (1992), 683.

7) P. Zhu and R. W. Smith: Acta Metall., 40 (1992), 3369.

8) Ch. A. Gardin and M. Rappaz: Acta Metall. Mater., 42 (1994), 2233.

9) Ch. A. Gardin, J. L. Desbiolles, M. Rappaz and Ph. Thevoz: Metall. Mater. Trans. A, 30A (1999), 3153.

10) C. Beckermann, H. J. Diepers, I. Steinbach, A. Karama and X. Tong: J. Computational Physics, 154 (1999), 468.

11) I. S. Cho and C. P. Hong: ISIJ Int., 37 (1997), 1098.

12) K. Y. Lee and C. P. Hong: ISIJ Int., 37 (1997), 38.

13) S. Y. Lee, S. M. Lee and C. P. Hong: ISIJ Int., 40 (2000), 48.

14) M. F. Zhu and C. P. Hong: ISIJ Int., 41 (2001), 436.

15) Ph.Thevoz: Sc. Dr. Thesis No. 765, Swiss Federal Inst. Tech., Lausanne, (1988).

16) W. C. Winegard, B. Chalmers: Trans. Am. Soc. Met., 46 (1954), 1214.

17) B. Chalmers: J. Aust. Inst. Metals, 8 (1963), 255.

18) K. A. Jackson, J. D. Hunt, D. R. Uhlmann and T. P. Seward: Trans. Metall. Soc. AIME, 236 (1966), 149.

19) R. T. Southin: Trans. Metall. Soc. AIME, 239 (1967), 220.

20) D. M. Smith, J. A. Eady, L. M. Hogan and D. W. Irwin: Metall. Trans. A, 22A (1991), 575.

21) F. K. Murakami, H. Aihara and T. Okamoto: Acta Metall., 32 (1984), 933.

22) H. Fredrikson, N. El Mahallawy, M. Taha, L. Xiang and G. Wanglow: Scand. J. Metall., 15 (1986), 127.

23) M. Rappaz and Ch. A. Gardin: Acta Metall. Mater., 41 (1993), 345.

24) W. Kruz, B. Giovanola and R. Trivedi: Acta Metall., 34 (1986), 823.

25) M. Rappaz and Ph. Thevoz: Acta Metall., 35 (1987), 1487.

26) W. A. Tiller and S. O'Hara: Proc. of the Solidification of Metals, ed. by H. Morrogh, The Iron and Steel Institute, Brighton, (1967), 27.

27) J. Hutt and D. H. StJohn: Int. J. Cast Metals Res., 11 (1998), 13. 